Review

\title{
Advances in radiotherapy and comprehensive treatment of high-grade glioma: immunotherapy and tumor-treating fields
}

\author{
Shiyu Liu1,2,3, Qin Zhao1,2,3, Weiyan Shi1,2,3, Zhuangzhuang Zheng1,2,3, Zijing Liu'1,2,3, Lingbin Meng4, Lihua \\ Dong1,2,3凶, Xin Jiang $1,2,3 \bowtie$ \\ 1. Department of Radiation Oncology, The First Hospital of Jilin University, Changchun 130021, China \\ 2. Jilin Provincial Key Laboratory of Radiation Oncology \& Therapy, The First Hospital of Jilin University, Changchun 130021, China \\ 3. NHC Key Laboratory of Radiobiology, School of Public Health, Jilin University, Changchun 130021, China \\ 4. Department of Hematology and Medical Oncology, Moffitt Cancer Center, Tampa, FL 33612, USA \\ $\triangle$ Corresponding authors: Prof. Lihua Dong, Ph.D, Department of Radiation Oncology, the First Hospital of Jilin University, 71 Xinmin Street, Changchun, \\ 130021 China; Phone: +86-15843073216; Email: 419522637@qq.com. Prof. Xin Jiang, Ph.D, Department of Radiation Oncology, the First Hospital of Jilin \\ University, 71 Xinmin Street, Changchun, 130021 ChinaPhone: +86-15804302750; email: jiangx@jlu.edu.cn
}

(1) The author(s). This is an open access article distributed under the terms of the Creative Commons Attribution License (https://creativecommons.org/licenses/by/4.0/). See http://ivyspring.com/terms for full terms and conditions.

Received: 2020.07.26; Accepted: 2020.11.21; Published: 2021.01.01

\begin{abstract}
High-grade gliomas (HGGs) are the most common primary malignant brain tumors. They have a high degree of malignancy and show invasive growth. The personal treatment plan for HGG is based on the patient's age, performance status, and degree of tumor invasion. The basic treatment plan for HGG involves tumor resection, radiotherapy (RT) with concomitant temozolomide (TMZ), and adjuvant TMZ chemotherapy. The basic radiation technology includes conventional RT, three-dimensional conformal RT, intensity-modulated RT, and stereotactic RT. As our understanding of tumor pathogenesis has deepened, so-called comprehensive treatment schemes have attracted attention. These combine RT with chemotherapy, molecular targeted therapy, immunotherapy, or tumor-treating fields. These emerging treatments are expected to improve the prospects of patients with HGG. In the present article, we review the recent advances in RT and comprehensive treatment for patients with newly diagnosed and recurrent HGG.
\end{abstract}

Key words: High-grade glioma; Immunotherapy; Molecular targeted drugs; Radiotherapy; Tumor-treating fields

\section{Introduction}

Glioma is the most common primary intracranial malignant tumor and accounts for $30 \%$ of all central nervous system tumors [1, 2]. The World Health Organization (WHO) classification of central nervous system tumors places gliomas into grades I-IV, with grades I and II indicating low-grade gliomas and grades III and IV, high-grade gliomas (HGGs) [3]. The incidence of HGGs is approximately 5 per 100,000 person-years. Researches have shown that gliomas are more common in adults and the elderly than in children; they also occur more often in men than in women [1, 2, 4, 5]. HGGs are characterized by the "three high and one low" principle: high incidence rate, high recurrence rate, high mortality, and low cure rate.

HGG accounts for 2.4 percent of all cancer deaths. Although clinicians and researchers have endeavored to improve treatment, prognosis remains poor [6]. After adjuvant chemotherapy, the 1-year survival rate of HGG patients is only $35 \%$ [7]. Researchers have carried out several studies using more precise radiotherapy (RT), but the effect is still unsatisfactory [8]. Therefore, new studies must be carried out to solve the difficulties associated with HGG treatment.

To treat newly diagnosed and recurrent HGG, RT generally involves conventional RT, three-dimensional conformal RT (3D-CRT), 
intensity-modulated RT (IMRT), and stereotactic RT (SRT). With a deeper understanding of the pathogenesis of HGG, a variety of comprehensive treatment modalities have been developed: RT has been combined with chemotherapy, molecular targeted therapy, and immunotherapy, while the tumor-treating fields (TTFields) approach has been combined with chemotherapy. These emerging treatment schemes can show better activity against tumors and improve survival rate. In this review, we focus on the progress of RT in the treatment of HGG.

\section{Radiotherapy}

\section{Conventional radiotherapy}

RT has a history of more than 100 years since its discovery in 1895, and it is now one of the three major treatment modalities for malignant tumors [9], some of which have such high sensitivity to radiation that use of RT alone is sufficient for cure. Examples of such tumors are nasopharyngeal carcinoma, early vocal fold cancer, and skin cancer. Conventional RT to treat HGG refers to two-dimensional conventional RT, which is applied to whole or part of the brain at an intensity of 45-60 Gy/25-30f [2, 10]. Shibamoto et al.[11] treated patients with HGG using conventional fractionation RT (64.8 Gy/36f) and demonstrated a median survival time of 14.5 months. Tanaka et al.[10] treated 60 patients using conventional RT (60 Gy/30f) and observed an overall survival (OS) of 12.4 months. They noted that radiation-induced white-matter abnormality was more frequent with high-dose RT than with conventional RT. In a clinical trial by Bleehen et al.[12], patients were divided into 45 Gy/20f and $60 \mathrm{~Gy} / 20 \mathrm{f}$ groups, with 1-year survival rates of $29 \%$ and $39 \%$, respectively. All these studies found that conventional RT showed good therapeutic effect in HGG, but the survival rate of patients following whole-brain irradiation was the same as that following tumor bed irradiation alone. So, irradiating whole brain is not necessary [13]. In summary, although conventional segmentation can achieve a treatment effect, it also leads to many side effects; thus, it is necessary to improve the RT technology to achieve a better treatment effect.

\section{Three-dimensional conformal radiotherapy}

3D-CRT applies a CT image to reconstruct the 3D structure of the tumor; the high-dose area is made coterminous with the lesion area to improve the radiation gain ratio and maximize the radiation dose within the lesion. Using 3D-CRT technology, tumor cells can be more effectively killed than when using conventional RT; furthermore, unnecessary irradiation of the surrounding normal tissues and organs can be reduced. Therefore, it is safe to increase the dose administered to the target tumor area, improve the local control rate of the tumor, improve survival, and reduce RT side effects. In the 1960s, Takahashi first proposed and clarified the basic concept and implementation method of conventional RT [14]. Proimos et al. [15] improved this method and applied it to clinical practice. In the 1990s, 3D-CRT technology became the leading technological modality for tumor treatment. Lee et al.[16] treated patients with HGG using 3D-CRT technology with a radiation dosage of $90 \mathrm{~Gy}$. However, 3D-CRT alone is not sufficient, even at very high doses, and its local control is still poor; thus, for treatment HGG, 3D-CRT is usually combined with TMZ. This approach shows improved efficacy and safety after surgery. Thibouw et al.[17] investigated patients with HGG who underwent 3D-CRT at a dosage of 40 Gy. They reported that the median survival was 13.4 months (range: 11.7-15.7 month). In conclusion, 3D-CRT is better than conventional RT, and 3D-CRT combined with TMZ confers more satisfactory results in HGG treatment.

\section{Intensity-modulated radiotherapy (IMRT)}

IMRT is a new type of external RT technology that has been widely applied in clinical treatment. Both IMRT and 3D-CRT are first-choice RT techniques to treat HGG; using these techniques, clinicians can control the shape of the radiation field and the intensity of the RT machine to ensure that the internal region of the target area corresponds with its surface. Both 3D-CRT and IMRT can reduce the radiation dose to surrounding normal tissues and organs as well as increase the radiation dose in the tumor area. These RT techniques aim to protect normal tissues and kill the tumor [18].

IMRT shows better target coverage than 3D-CRT, and the dose required is lower when the tumor has a complex or irregular shape. In addition, it can reduce neurological toxicity as well as the dose applied to the optic nerve and other normal tissues [17, 19]. Hermanto et al. [20] compared patients treated using 3D-CRT and IMRT. They found that the total integral dose on the normal tissues was reduced by $7 \%-10 \%$ using IMRT, that IMRT completed DNA breakage in tumor cells to promote cell death, and that it did not increase the dose or volume of normal tissue exposed to low doses of radiation. Cho et al. [21] treated HGG using simultaneous, integratedboost IMRT (SIB-IMRT), with the following radiation dose: gross target volume, $60 \mathrm{~Gy}$; clinical target volume, $50 \mathrm{~Gy}$. The results showed that the median survival time was 14.8 months and the progression-free survival (PFS) time was 11 months. Thilmann et al. [22] treated HGG using IMRT and 
found that the treatment delivered a high dose to the enhancing lesion, and that the overall does was identical to that of an equivalent CTV dose delivered at the same time. Many clinical studies have shown that OS and PFS are higher in the IMRT group than in the conventional RT group, that treatment of HGG using low-segmentation and simultaneous IMRT shows satisfactory therapeutic effects, and that patients treated using this approach display good tolerance of adverse reactions. Importantly, IMRT has developed into a widely used RT technology; in fact, it is usually the first-choice RT in the treatment of HGG.

\section{Hyper-fractionated radiotherapy}

Hyper-fractionated radiotherapy (HFRT) is a radiation technique that refers to reducing the dose of each irradiation twice a day or more, using a large number of smaller doses to make the total dose higher than the conventional dose. HFRT can improve the sublethal damage repair, has less dependence on oxygen, and increases the radiation opportunity of tumor cells in the more sensitive stage of the cell cycle [23]. Prados et al. [24] treated HGG patients with 1.6 Gy each time, twice a day, and the total dose was 70.4 Gy. The results revealed that median survival period was 40 weeks and PFS period was 19 weeks. Nelson et al.[23] treated HGG patients with 64.8, 72, 76.8, and $81.6 \mathrm{~Gy}$, respectively, and observed that a total dose of 72 Gy/60f showed the best effect. Hasegawa et al. [25] transplanted xenografts of a human malignant glioma into nude mice and irradiated then with hyper-fractionated $24 \mathrm{~Gy} / 20 \mathrm{f}$ (two fractions per day), which had a good effect on eliminating the tumor. Ali et al. [26] compared the patients who received conventional RT and HFRT. The results showed that the median survival time was 11.3 and 13.1 months, respectively. Xin et al. [27] conducted a comparative experiment and found that there was no clear difference in OS and PFS between the participants receiving HFRT and those receiving conventional RT. Although the dose administered in HFRT is higher than that given in conventional RT, there was no significant difference between the side effects and overall cognitive ability [28]. Therefore, HFRT is not a necessary treatment technique for HGG.

\section{Stereotactic radiotherapy (SRT)}

SRT uses stereotactic and multi-center, rotating irradiation technology to focus high-energy radiation in 3D space onto the limited target area of the lesion. It is characterized by high dose to the target area, large dose gradients with the surrounding normal tissues, and rapid tapering of radiation dose. However, SRT cannot cover the same area as conventional RT, so it cannot clear cells at the edge of the tumor and is therefore unsuitable as an initial treatment. Nonetheless, it is associated with a reduced reaction of the normal brain tissue to radiation, as well as with a decrease in complications, although it can cause vascular embolism, the death of proliferating cells, and marked necrosis within the treatment area. SRT has certain survival benefits for HGG patients with minimal disease burden [29]. Shrieve et al.[30] treated patients with HGG using SRT. They reported that the 12 - and 24 month-OS rates were $88.5 \%$ and $35.9 \%$, respectively, demonstrating that SRT has a survival advantage over other kinds of RT. Reynaud et al.[31] investigated survival outcomes and safety in patients with recurrent HGG using HFSRT. They reported that HFSRT is feasible, with minimal adverse effects and a median OS of 15.6 months. In recent years, gamma knife stereotactic surgery has been applied in the rescue treatment of patients with recurrent HGG. This approach can improve the survival rate and reduce the burden [32]. Overall, SRT should be considered for the treatment of recurrent HGG as it can reduce the treatment volume and decrease treatment-related toxicity, thus improving the safety of reirradiation [33].

\section{Combination therapy}

\section{RT combined with chemotherapy}

Chemotherapy can reduce the volume of lesions, improve local blood circulation, and increase radiosensitivity. Chemotherapy drugs have cell cyclespecific cytotoxic effects on S-phase cells, while concurrent RT and chemotherapy have complementary effects on cell killing. The most frequently used chemotherapy drug in HGG treatment is oral TMZ, which is widely distributed throughout the body without passing through the liver [6]. It localizes easily to brain tumor cells after passing through the blood-brain barrier, and it acts on all cells in all stages of the cell cycle. Also, it shows rapid absorption and has a low incidence of side effects when administered orally [34].

The DNA repair enzyme $\mathrm{O}^{6}$-methylguanineDNA methyltransferase (MGMT) inhibits the killing of tumor cells by TMZ. Methylation of the MGMT promoter silences this gene in cancer so that the cells no longer produce MGMT. This phenomenon is associated with tumor regression as well as with prolonged OS and disease-free survival $[35,36]$. The basic treatment regimen is to administer TMZ concurrently with radiation for 6 weeks, followed by 6 months of adjuvant TMZ therapy. However, because TMZ shows fair tolerability and because no effective second-line therapies are available, up to 12 cycles of treatment are now allowed. Roldan et al. [37] 
compared a treatment regimen of six cycles of monthly adjuvant TMZ with regimens involving more than six cycles. They found that the latter conferred significant benefits in terms of both PFS and OS. Hart et al. [38] conducted a randomized controlled trial and showed that TMZ increased both OS and PFS, but it was found to be associated with a significant impact on quality of life and increased the risk of hematological complications, fatigue, and infection. Stupp et al. [6] observed that patients with HGG treated using RT plus TMZ showed a 37\% reduction in the risk of death compared with those who received RT alone. Jaymin et al. [39] treated one group of patients with HGG using chemotherapy alone and another group using chemotherapy plus conventional RT. They demonstrated no OS difference between the chemotherapy-alone and conventional RT groups. In elderly patients ( $\geq 65$ years old) with newly diagnosed HGG, the addition of TMZ to short-course RT resulted in longer survival than short-course RT alone [40]. Similarly, another chemotherapy treatment regimen is also available: procarbazine, lomustine, and vincristine (PCV). In several studies, PFS, but not OS, was better in patients with HGG treated using RT plus PCV than in those treated using RT alone, suggesting that chemotherapy has a delayed benefit $[41,42]$. One randomized trial revealed that primary chemotherapy was not superior to primary RT among patients with different kinds of glioma [43]. Jakacki et al. [44] compared the survival rate between patients with glioma treated using lomustine plus TMZ and those treated using TMZ alone after conventional RT. They concluded that the addition of lomustine to TMZ as adjuvant therapy was associated with significantly better outcome. In our previous research, we concluded that RT plus chemotherapy is associated with considerable toxicity [45]. In addition, RT can lead to secondary gliomas, and so other researchers have also considered combination therapy a potential avenue of treatment for radiation-induced gliomas [46]. Overall, the combination of RT and chemotherapy is now the first choice of treatment for HGG.

\section{Molecular targeted drugs combined with RT}

Currently, because glioma is resistant to RT and chemotherapy, it has a high recurrence rate and the treatment effect is not satisfying. To overcome these limitations of treatment, molecular targeted drugs combined with RT and chemotherapy have been researched. Molecular targeted drugs initiate specific molecular signal transduction pathways to kill the tumor effectively.

Molecular targeted therapies include vascular endothelial growth factor (VEGF) inhibitors and epidermal growth factor receptor (EGFR) inhibitors combined with RT $[37,47,48]$. The signal transduction pathways involved may activate apoptosis, inhibit the cell cycle, or block vascular growth [49]. Bevacizumab is a common molecular targeted drug; it is a humanized monoclonal antibody against VEGF [50] that can activate the VEGF downstream pathway (RAS-RAF-MAPK, etc.) through protein phosphorylation. In this manner, VEGF plays an important role in the growth and development of tumor cells [51]. Bevacizumab is currently approved to treat patients with recurrent HGG, but not as part of the upfront regimen for newly diagnosed HGG [52]. Friedman et al. [53] treated patients with HGG using $10 \mathrm{mg} / \mathrm{kg}$ bevacizumab, either alone or in combination with irinotecan. Both of these regimens produced satisfactory results in recurrent glioma. Hasselbalch et al. [54] observed that combining cetuximab with both bevacizumab and irinotecan was safe and effective in patients with recurrent HGG, that the median PFS was 16 weeks, and that the median OS was 30 weeks, which was an encouraging response rate. To treat patients with recurrent HGG, the combination of the anti-programmed cell death 1 (PD-1) monoclonal antibody nivolumab and bevacizumab showed no better efficacy than bevacizumab alone [55, 56]. Sathornsumetee et al.[57] demonstrated that bevacizumab combined with the EGFR tyrosine kinase inhibitor erlotinib was adequately tolerated in patients with recurrent HGG. Bovi et al. [58] compared PFS between two treatment strategies treating recurrent HGG patients and found that bevacizumab combined with RT, PFS was 12 months compared to bevacizumab alone where PFS was 4 months. Westphal et al. [37] conducted a clinical trial that failed to show any significant advantage of nimotuzumab in terms of prolonging PFS and OS when combined with bevacizumab to treat patients with HGG. Hofmann et al. [59] also utilized bevacizumab, citing improved survival in patients with recurrent HGG compared to standard therapy without bevacizumab (median OS, 10.3 vs. 4.2 months, $p=0.023$ ). In a word, bevacizumab could improve clinical outcomes and the patient's quality of life in HGG treatment and show improvement in newly diagnosed and recurrent HGG patients.

Gefitinib is a potent small molecule inhibitor of EGFR tyrosine kinase. Schwer et al. [60] treated recurrent HGG using SRT combined with gefitinib and reported that a dose of $36 \mathrm{~Gy}$ in three fractions was well tolerated, with gefitinib at a daily dose of 250 $\mathrm{mg}$. Aflibercept is a recombinant fusion protein of the VEGF extracellular domains. It binds with high affinity and can also scavenge VEGF [61]. Groot et al. [62] evaluated its treatment efficacy in patients with 
recurrent HGG. They found that aflibercept monotherapy was not as effective as bevacizumab and that it had moderate toxicity. Bastiien et al.[63] summarized some possible reasons for the failure of this molecular targeted therapy, which are as follows: (1) The targeted molecular pathways may have built-in redundancies; (2) It may be difficult to penetrate the blood-brain barrier to the central nervous system while avoiding neurotoxicity. Overall, RT combined with chemotherapy and molecular targeted drugs can lead to better treatment outcomes than the use of RT alone. This approach has become increasingly popular to treat recurrent HGG.

\section{Immunotherapy}

Nowadays, immunotherapy is being applied to a broader range of diseases, and research is deepening our understanding of how immunotherapy could be used. The immune system can protect the host and inhibit the tumor microenvironment [64]. Immunotherapy induces antitumor responses via the host immune system to clear tumors [65]. RT releases tumor antigens and modulates immunological pathways, leading to increased tumor antigen concentration and major histocompatibility complex (MHC) on the tumor cell surface and priming of tumor-specific cytotoxic $\mathrm{T}$ cells, ultimately resulting in the immunogenic death of tumor cells $[55,66,67]$. The primary target for the T-cell receptor is MHC loaded with tumor antigen [67]. MHC I participates in antigen recognition by $\mathrm{CD} 8{ }^{+}$cytotoxic $\mathrm{T}$ lymphocytes (CTLs), while MHC II participates in antigen recognition by $\mathrm{CD}^{+} \mathrm{Th}$ cell. The mechanisms of immunotherapy are depicted in Figure 1 [68].

In recent years, antigen-specific cancer vaccines and immune checkpoint blockers has provided promising immunotherapeutic approaches to treat HGG [69]. Two immune checkpoints being studied are cytotoxic T-lymphocyte-associated protein 4 (CTLA-4) and PD-1 [70, 71], which along with programmed death-ligand 1 (PD-L1) is widely expressed in patients with either primary or recurrent HGG. The binding of PD-1 to PD-L1 induces apoptosis or leads to exhaustion of activated immune cells; therefore, blocking this interaction enhances antitumor activity, so patients can be treated using PD-1 and PD-L1 checkpoint inhibitors [72, 73]. Through interactions with co-stimulatory molecules on other cells, CTLA-4 acts to decrease T-cell responsiveness [74]. Tumor-associated macrophages (TAMs) contribute to tumor growth, metastasis, and neovascularization. In tumors trending towards malignancy, TAMs stimulate blood vessels and suppress antitumor immunity. HGG is characterized by extensive neo-angiogenesis, so TAMs are closely related to the pathogenesis of HGG $[75,76]$. Chimeric

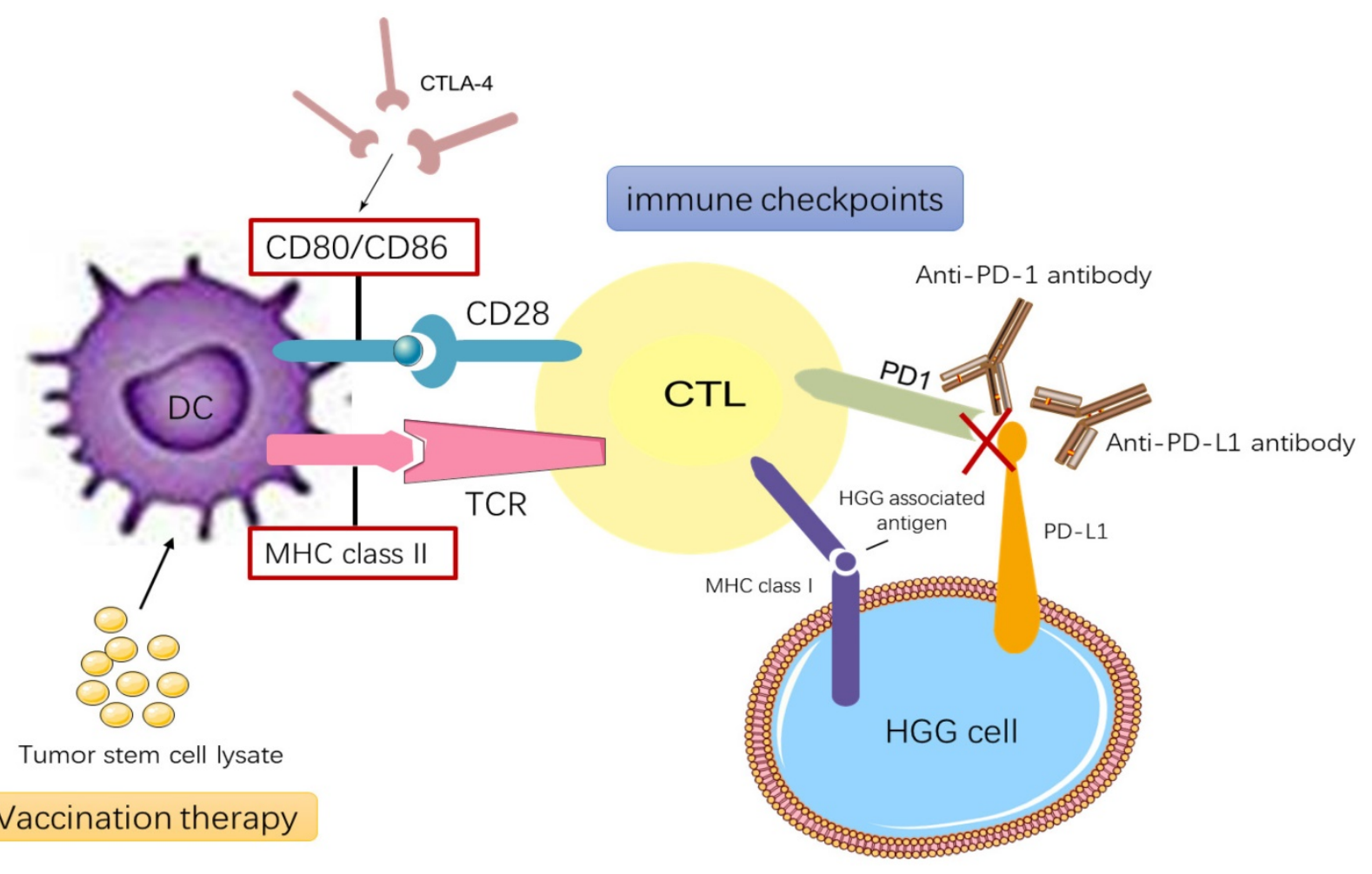

Figure 1. Current immunotherapy modalities for HGG treatments. HGG vaccination therapy relies on DC stimulated by tumor stem cell lysate or the interaction between MHC class II-TCR and CD80 and/or CD86-CD28. CTLs activate and destroy cells containing glioblastoma- associated antigens presented on MHC class I molecules. HGG cells upregulate the PD- L1 that combines with complementary receptors on the CTLs to cause inhibition of lymphocyte activation. CTLA-4 suppresses immune- checkpoint molecule that binds CD80 and CD86 and prevents their interaction with CD28. 
antigen receptor- $\mathrm{T}$ cell therapy integrates the target antigen receptor into the normal $\mathrm{T}$ cell gene through the carrier, while the target effector $\mathrm{T}$ cell is applied to the tumor site as an anti-tumor treatment.[77] Scheetz et al.[69] have developed a personalized nano-disc vaccine loaded with $\mathrm{CpG}-\mathrm{a}$ toll-like receptor 9 agonist-as well as tumor-specific neoantigens that can be used in combination immunotherapy to treat recurrent gliomas. Zeng et al. [78] studied the efficacy of combination treatment using the anti-PD-1 antibody and RT in HGG mouse models. They demonstrated that this combination therapy resulted in long-term survival. Preclinical evidence suggests that hyper-fractionation RT can stimulate the immune system and make immunotherapy more effective [71]. One mechanism accounting for the enhanced effectiveness of combinatorial treatment radiation-induced inflammation results in PD-L1 upregulation in cancer cells, macrophages, and dendritic cells [79]. Fractionated RT leads to upregulation of PD-L1 expression in tumor cells, which generates therapeutic immune responses that can reduce tumor burden and improve survival [80]. In conclusion, many studies have shown that immunotherapy can confer satisfactory therapeutic effects, especially when combined with molecular targeted therapy (Table 2).

\section{Tumor-treating fields (TTFields)}

TTFields are an antimitotic treatment modality delivered via low-intensity, intermediate-frequency (200 kHz) alternating electric fields ( $\geq 18$ hours/day) using four insulated transducer arrays placed directly on the skin in the region surrounding the tumor
[81-84]. Through dipole redistribution, this treatment acts on cells in the middle and late stages of mitosis $\left(G_{1} / S\right.$ or $\left.G_{2} / M\right)$, hinders the orderly arrangement and location of tubulin, disrupts the normal assembly of spindle microtubules, and leads to breakage of the centromere and damages the organelle structure[84]. The result is an overall decline in cell proliferation followed by apoptosis, which is strongly affected by mutations in p53. TTFields act on several molecular targets/pathways to influence $\mathrm{Ca}^{2+}$ and electrical signals. One recent study demonstrated that voltage-gated $\mathrm{Ca}^{2+}$ channel activity contributes to the cellular stress response to TTFields and that voltage-gated $\mathrm{Ca}^{2+}$ channel inhibition may augment the effects of TTFields [85]. It thus follows that TTFields can reduce cell proliferation by specifically interfering with key proteins involved in cell division, leading to mitotic mutations and subsequent cell death [86].

The mechanisms of TTFields are depicted in Figure 2. TTFields can delay the repair of DNA damage caused in tumor cells by RT and chemotherapy, thus playing a role in killing tumor cells when used in coordination with RT and chemotherapy. This technique downregulates the $B R C A / F A N C$ genes and increases DNA single-/double-strand damage, thus destroying the DNA replication and repair mechanism in tumor cells. Because they act on hypoxic cells, TTFields inhibit mitochondrial autophagy in tumor cells, increase the production of reactive oxygen species, and improve the oxygen sensitivity.

Table 1. Clinical trials of IMRT in HGG

\begin{tabular}{lllll}
\hline Author & Total Dose $(\mathrm{Gy})$ & Plan & Number of Patients & Result \\
\hline Sutera et al 2019 & $60 / 30 \mathrm{f}$ & IMRT & 291 & OS:14.2mo \\
Carlson et al 2015 & PTV1:60Gy/10f PTV2:30Gy/10f & IMRT+TMZ+BEV & 30 & PFS:12.8mo OS:16.3mo \\
Carlson et al ${ }^{2015}$ & PTV1:60Gy/10f PTV2:30Gy/10f & IMRT+TMZ & 26 & PFS:9.4mo OS:16.3mo \\
Zhong et al ${ }^{2019}$ & $64 / 27 \mathrm{f}$ & IMRT+TMZ & 80 & PFS:15mo OS:21mo \\
Reddy et al2012 & $60 \mathrm{~Gy} / 10 \mathrm{f}$ & IMRT+TMZ & 24 & OS:16.6mo \\
Monjazeb et al ${ }^{2012}$ & $70 \mathrm{~Gy} / 28 \mathrm{f}$ & IMRT & 21 & PFS:6.5mo OS: $13.6 \mathrm{mo}$ \\
Jastaniyah et al ${ }^{2013}$ & $54.4 \mathrm{~Gy} / 20 \mathrm{f}$ & IMRT+TMZ & 25 & OS:15.67mo PFS:6.7mo \\
\hline
\end{tabular}

Table 2. Clinical trials of immunotherapy in HGG

\begin{tabular}{|c|c|c|c|c|c|}
\hline Author & Target & Number of patients & Vaccine & Result & Reference \\
\hline \multirow[t]{2}{*}{ Cloughesy et al2019 } & PD1 & 16 & neoadjuvant pembrolizumab & PFS:99.5d & {$[101]$} \\
\hline & PD1 & 16 & adjuvant nivolumab & PFS:72.5d & \\
\hline \multirow[t]{2}{*}{ Desjardins et al 2018} & CD155 & 61 & PVSRIPO & OS: $12.5 \mathrm{mo}$ & [102] \\
\hline & -- & 104 & -- & OS:11.3mo & \\
\hline Brown et al2016 & IL13Ra2 & 1 & IL13BB $\zeta-C A R T$ & cytokines and immune cells in the cerebrospinal fluid increase & [103] \\
\hline O'Rourke et al ${ }^{2017}$ & EGFR & 10 & CART-EGFRvIII & OS:251d & {$[104]$} \\
\hline Hilf et $\mathrm{al}^{2019}$ & $\mathrm{CD} 4 / \mathrm{CD} 8$ & 16 & APVAC1/APVAC2 & OS:29mo PFS:14.2mo & [105] \\
\hline
\end{tabular}




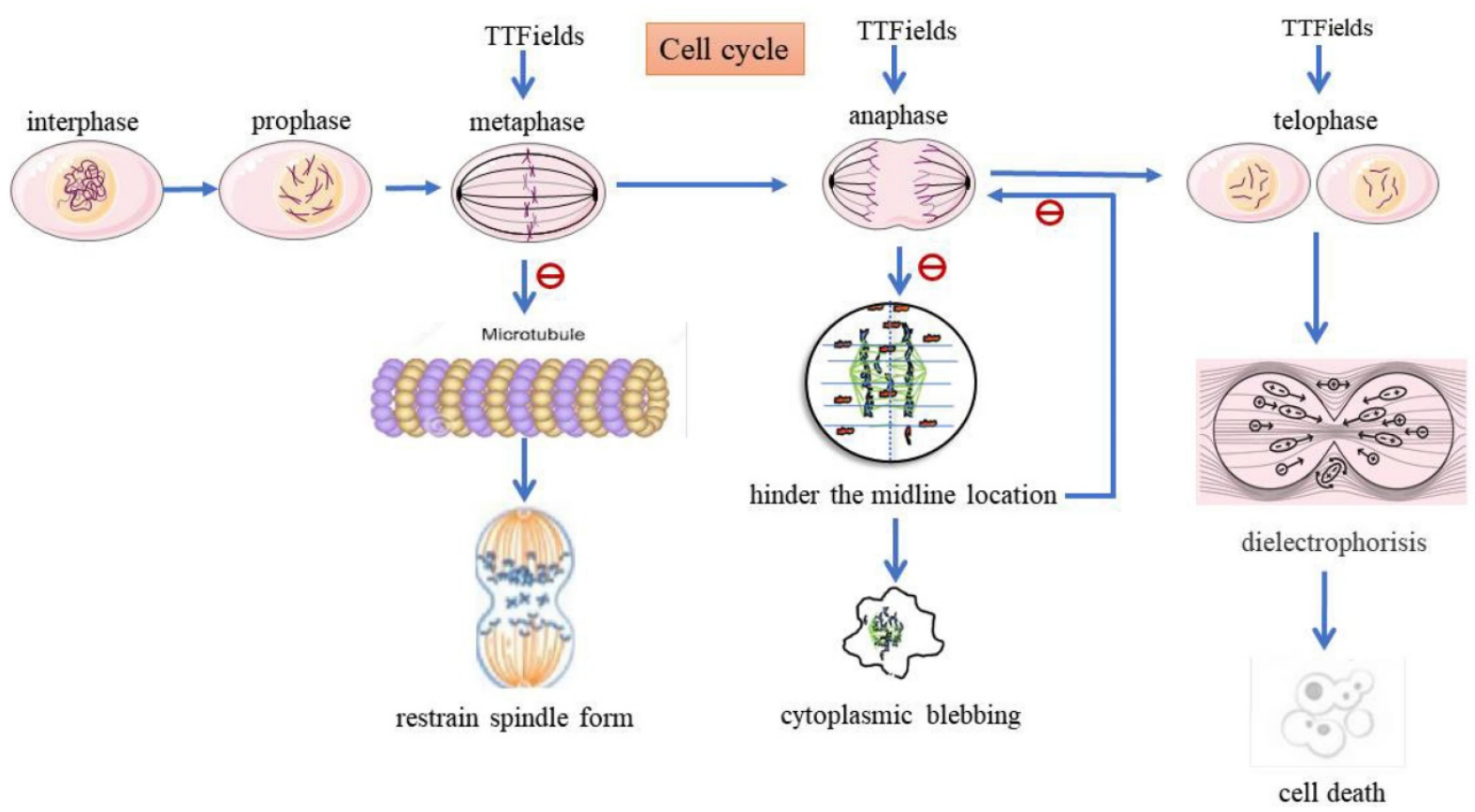

Figure 2. The mechanisms of TTFields treating HGG. TTFields will interfere with appropriate microtubules (MT) to prevent mitosis and spindle formation. TTFields exert directional force on MT, resulting in abnormal spindle formation, followed by stagnation or delay of mitosis. It may be caused by improper connection between chromosomes and spindle. TTFields suppress regulated BRCA / FANC gene, which make DNA replication fork pause and break down, and it can increase DNA damage and destroy DNA replication and repair mechanism of HGG cells.

Table 3. Summary of studies for recurrent HGG treated with TTFields

\begin{tabular}{|c|c|c|c|c|c|}
\hline Authors & Number of patients & Arms & OS (mo) & PFS (mo) & reference \\
\hline Lazaridis et al 2020 & 16 & TTFields+TMZ +lomustine & -- & 20 & [91] \\
\hline Toms et al 2019 & 695 & TTFields+TMZ & 24.9 & 8.2 & [106] \\
\hline \multirow[t]{2}{*}{ Stupp et $\mathrm{al}^{2017}$} & 466 & TTFfields + TMZ ( $150-200 \mathrm{mg} / \mathrm{m}^{2} / \mathrm{d}$ for 5 days every 28 days for 6 cycles) & 20.9 & 6.7 & [82] \\
\hline & 229 & TMZ alone & 16 & 4 & [82] \\
\hline Kirson et al ${ }^{2007}$ & 10 & TTFields (200kHZ, 2 V/cm,6d) & 15.5 & 6.525 & [107] \\
\hline \multirow[t]{2}{*}{ Kirson et al ${ }^{2009}$} & 10 & TTFields $(200 \mathrm{kHZ}, 1.75 \mathrm{~V} / \mathrm{cm}, 3 \mathrm{~d})+\mathrm{TMZ}$ & $>39$ & 38.75 & [108] \\
\hline & 10 & $\mathrm{TMZ}$ & $>14.7$ & 7.75 & \\
\hline \multirow[t]{2}{*}{ Stupp et $\mathrm{al}^{2015}$} & 210 & TTFields+TMZ (150-200 mg/m²/d) & 20.5 & 7.1 & [109] \\
\hline & 105 & TMZ & 15.6 & 4 & \\
\hline Mrugala et al ${ }^{2014}$ & 457 & NovoTTF & 9.6 & 4.1 & [110] \\
\hline \multirow[t]{2}{*}{ Stupp et al ${ }^{2012}$} & 117 & TTFields (NovoTTF-100A) & 6.6 & 2.2 & [111] \\
\hline & 120 & TMZ & 6 & 2.1 & \\
\hline \multirow[t]{2}{*}{ Ballo et al 2019} & 148 & TTFields(LMiFI >1.06 V/cm) & 24.3 & 8.1 & {$[88]$} \\
\hline & 192 & TTFields(LMiFI<1.06 V/cm) & 21.6 & 7.9 & \\
\hline \multirow[t]{2}{*}{ Lu et al 2019} & 18 & TMZ+bevacizumab+irinotecan+TTFields & 18.9 & 10.7 & [92] \\
\hline & 30 & bevacizumab +TTFields & 11.8 & 4.7 & \\
\hline
\end{tabular}

When using TTFields, clinicians should adjust the transducer array layout according to the specific tumor location to improve the field strength and achieve the best effect of tumor cutting [87]. And different TTFields intensities receive different results in PFS and OS for HGG patients [88]. Many clinical trials have been carried out comparing TTFields plus TMZ with TMZ alone, and some trial has added lomustine to the treatment regimen, as shown in Table 3. Stupp et al. [82] treated patients with HGG using either TTFields plus TMZ or TMZ alone. They found that the median OS was 20.9 months and 16.0 months, respectively. The addition of TTFields to standard TMZ treatment in patients with HGG resulted in improved survival, with no negative impact on health-related quality of life other than itchy skin [89, 90]. Furthermore, Lazaridis et al. [91]suggested that TTFields combined with lomustine and temozolomide was superior to TTFields combined with temozolomide monotherapy in patients with HGG. And this analysis provided first indication that the combination of TTFields/lomustine/temozolomide was safe and feasible. Lu et al. [92]compared PFS and OS between the two treatment strategies and found that the combination of TTFields, TMZ, bevacizumab, and irinotecan might play a more important role in the improvement of PFS and OS among HGG patients than the combination of TTFields and bevacizumab. Overall, TTFields combined with chemotherapy can prolong PFS and OS and is therefore the new pattern 
of treatment for patients with newly diagnosed or recurrent HGG $[85,93,94]$.

\section{Conclusion}

Considerable progress has been made in the field of RT in recent years, and it is important that clinicians and researchers maximize the survival benefits afforded by this treatment modality. At present, HGG is mainly treated using surgical resection combined with chemotherapy, RT, immunotherapy, and other comprehensive treatments. Postoperative external beam RT with concomitant TMZ and adjuvant TMZ chemotherapy has been recommended as a standard treatment for newly diagnosed HGG in adults. The typical RT dose is 60 Gy divided into 30 fractions. RT should be started as early as possible (2-6 weeks after surgery), and it can effectively prolong the survival period. The therapeutic effect of RT is cumulative, so it would not be effective if delivered all at one time. Instead, it takes effect if the patient accumulates a certain dose of radiation. Therefore, the exact irradiation time is not very strict-RT can be started as soon as possible provided a patient is prepared and a treatment plan has been established. In addition, age, pathological grade, and chemotherapy are related to the prognosis of HGG. Higher age is associated with a higher risk of death. There is an improved tend of prognosis with younger HGG patients. In addition, patients with low-grade gliomas have a better prognosis than those with HGG, and glioblastomas have the worst prognosis. Furthermore, postoperative concurrent chemoradiotherapy will further eliminate the tumor or inhibit the growth of the tumor, which will also improve the survival rate of HGG patients. Although these therapies represent a significant accomplishment, the treatment course is still riddled with numerous obstacles and challenges. RT may increase the risk of neurocognitive side effects in the long term, and the prognosis of HGG is still poor, so researchers face many challenges. As technology progresses, more exploration and research will be conducted, and more advanced technologies and approaches will be applied to the treatment of HGG in future. Furthermore, the diagnosis of HGG will become more accurate, and the therapeutic effect will be more refined.

\section{Abbreviations}

CIRT: Carbon ion radiotherapy; CRT: Conventional radiotherapy; CTLA-4: Cytotoxic T-lymphocyte-associated protein 4; CTV: Clinical Target Volume; DC: Dendritic cell; EGFR : Epidermal growth factor receptor; GTV: Gross Target Volume; HFSRT: hypofractioned stereotactic radiotherapy;
HGG: High-grade gliomas; IMRT: Intensity-modulated radiotherapy; LET: Linear energy transfer; MT: Microtubules; mTOR: mammalian Target of rapamycin; NCCN: National Comprehensive Cancer Network; OS: Overall survival; RBE: Relative biological effectiveness; PBT: Proton beam therapy; PD-1: Programmed cell death-1; PD-L1: Programmed death-ligand 1; PFS: Progress-free survival; PVC: Procarbazine, lomustine, and vincristine; SIB-IMRT: Simultaneous integrated boost intensity modulated radiotherapy; SRT: Stereotactic radiotherapy; TAMs: Tumor-associated macrophages; TGF- $\beta$ : Transforming growth factor- $\beta$; TMZ: Temozolomide; TTFields: Tumor-treating fields; VEGF: Vascular endothelial growth factor; WHO: World Health Organization; 3D-CRT: three-dimensional conformal radiotherapy; 2D-CRT: two-dimensional conventional radiotherapy.

\section{Acknowledgements}

We would like to thank Editage (www.editage.cn) for English language editing.

\section{Funding Statement}

This work was supported by the National Natural Science Foundation of China (Grant number 81570344); National Key R\&D Program of China (Grant number 2017YFC0112100); the Education Department Foundation of Jilin Province (Grant number JJKH20201036KJ); the Health and Family Planning Commission of Jilin Province Foundations (Grant number 2016Q034 and 2017J11) and the Jilin Provincial Science and Technology Foundations (Grant number 20180414039GH and 20190201200JC).

\section{Author Contributions}

Conceptualization, X.J. and LH.D.; software, investigation, WY.S. and Q.ZH.; resources, ZZ.ZH.; writing-original draft preparation, SY.L., ZJ.L., Q.ZH., and LB.M.; writing-review and editing, L.B.M., LH.D., and X.J.; funding acquisition, X.J. All authors read and approved the manuscript.

\section{Competing Interests}

The authors have declared that no competing interest exists.

\section{References}

1. Sadik ZHA, Hanssens PEJ, Verheul JB, Beute GN, Te Lie S, Leenstra S, et al. Gamma knife radiosurgery for recurrent gliomas. J Neurooncol. 2018; 140: 615-22.

2. Khan L, Soliman H, Sahgal A, Perry J, Xu W, Tsao MN. External beam radiation dose escalation for high grade glioma. Cochrane Database Syst Rev. 2016: Cd011475.

3. Louis DN, Ohgaki H, Wiestler OD, Cavenee WK, Burger PC, Jouvet A, et al. The 2007 WHO classification of tumours of the central nervous system. Acta Neuropathol. 2007; 114: 97-109.

4. Larjavaara S, Mantyla R, Salminen T, Haapasalo H, Raitanen J, Jaaskelainen J, et al. Incidence of gliomas by anatomic location. Neuro Oncol. 2007; 9: 319-25. 
5. Gousias K, Markou M, Voulgaris S, Goussia A, Voulgari P, Bai M, et al. Descriptive epidemiology of cerebral gliomas in northwest Greece and study of potential predisposing factors, 2005-2007. Neuroepidemiology. 2009; 33: 89-95.

6. Stupp R, Mason WP, van den Bent MJ, Weller M, Fisher B, Taphoorn MJ, et al. Radiotherapy plus concomitant and adjuvant temozolomide for glioblastoma. N Engl J Med. 2005; 352: 987-96.

7. Stupp R, Hegi ME, Mason WP, van den Bent MJ, Taphoorn MJ, Janzer RC, et al. Effects of radiotherapy with concomitant and adjuvant temozolomide versus radiotherapy alone on survival in glioblastoma in a randomised phase III study: 5-year analysis of the EORTC-NCIC trial. Lancet Oncol. 2009; 10: 459-66.

8. Badiyan SN, Markovina S, Simpson JR, Robinson CG, DeWees T, Tran DD, et al. Radiation therapy dose escalation for glioblastoma multiforme in the era of temozolomide. Int J Radiat Oncol Biol Phys. 2014; 90: 877-85.

9. Yin W, Chen B, Tian F, Yu Y, Kong FM. The growth of radiation oncology in mainland China during the last 10 years. International journal of radiation oncology, biology, physics. 2008; 70: 795-8.

10. Tanaka M, Ino Y, Nakagawa K, Tago M, Todo T. High-dose conformal radiotherapy for supratentorial malignant glioma: a historical comparison. Lancet Oncol. 2005; 6: 953-60.

11. Shibamoto Y, Nishimura Y, Tsutsui K, Sasai K, Takahashi M, Abe M. Comparison of accelerated hyperfractionated radiotherapy and conventional radiotherapy for supratentorial malignant glioma. Japanese journal of clinical oncology. 1997; 27: 31-6.

12. Bleehen NM, Stenning SP. A Medical Research Council trial of two radiotherapy doses in the treatment of grades 3 and 4 astrocytoma. The Medical Research Council Brain Tumour Working Party. British journal of cancer. 1991; 64: 769-74.

13. Garden AS, Maor MH, Yung WK, Bruner JM, Woo SY, Moser RP, et al. Outcome and patterns of failure following limited-volume irradiation for malignant astrocytomas. Radiotherapy and oncology : journal of the European Society for Therapeutic Radiology and Oncology. 1991; 20: 99-110.

14. Takahashi S. Conformation radiotherapy. Rotation techniques as applied to radiography and radiotherapy of cancer. Acta radiologica: diagnosis. 1965: Suppl 242:1+

15. Proimos BS. Beam-shapers oriented by gravity in rotational therapy. Radiology. 1966; 87: 928-32 passim.

16. Chan JL, Lee SW, Fraass BA, Normolle DP, Greenberg HS, Junck LR, et al. Survival and failure patterns of high-grade gliomas after three-dimensional conformal radiotherapy. J Clin Oncol. 2002; 20: 1635-42.

17. Thibouw D, Truc G, Bertaut A, Chevalier C, Aubignac L, Mirjolet C. Clinical and dosimetric study of radiotherapy for glioblastoma: three-dimensional conformal radiotherapy versus intensity-modulated radiotherapy. J Neurooncol. 2018; 137: 429-38.

18. Chen C, Damek D, Gaspar LE, Waziri A, Lillehei K, Kleinschmidt-DeMasters $\mathrm{BK}$, et al. Phase I trial of hypofractionated intensity-modulated radiotherapy with temozolomide chemotherapy for patients with newly diagnosed glioblastoma multiforme. Int J Radiat Oncol Biol Phys. 2011; 81: 1066-74.

19. Suzuki M, Nakamatsu K, Kanamori S, Okumra M, Uchiyama T, Akai F, et al. Feasibility study of the simultaneous integrated boost (SIB) method for malignant gliomas using intensity-modulated radiotherapy (IMRT). Japanese journal of clinical oncology. 2003; 33: 271-7.

20. Hermanto U, Frija EK, Lii MJ, Chang EL, Mahajan A, Woo SY. Intensity-modulated radiotherapy (IMRT) and conventional three-dimensional conformal radiotherapy for high-grade gliomas: does IMRT increase the integral dose to normal brain? Int J Radiat Oncol Biol Phys. 2007; 67: $1135-44$

21. Cho KH, Kim JY, Lee SH, Yoo H, Shin SH, Moon SH, et al. Simultaneous integrated boost intensity-modulated radiotherapy in patients with high-grade gliomas. Int J Radiat Oncol Biol Phys. 2010; 78: 390-7.

22. Thilmann $\mathrm{C}$, Zabel A, Grosser $\mathrm{KH}$, Hoess A, Wannenmacher $\mathrm{M}$, Debus J. Intensity-modulated radiotherapy with an integrated boost to the macroscopic tumor volume in the treatment of high-grade gliomas. Int J Cancer. 2001; 96: $341-9$

23. Laperriere N, Zuraw L, Cairncross G, Cancer Care Ontario Practice Guidelines Initiative Neuro-Oncology Disease Site G. Radiotherapy for newly diagnosed malignant glioma in adults: a systematic review. Radiother Oncol. 2002; 64: 259-73.

24. Prados MD, Wara WM, Sneed PK, McDermott M, Chang SM, Rabbitt J, et al. Phase III trial of accelerated hyperfractionation with or without difluromethylornithine (DFMO) versus standard fractionated radiotherapy with or without DFMO for newly diagnosed patients with glioblastoma multiforme. Int J Radiat Oncol Biol Phys. 2001; 49: 71-7.

25. Hasegawa M, Niibe H, Mitsuhashi N, Yamakawa M, Kato S, Furuta M, et al. Hyperfractionated and hypofractionated radiation therapy for human malignant glioma xenograft in nude mice. Jpn J Cancer Res. 1995; 86: 879-84.

26. Ali AN, Zhang P, Yung WKA, Chen Y, Movsas B, Urtasun RC, et al. NRG oncology RTOG 9006: a phase III randomized trial of hyperfractionated radiotherapy (RT) and BCNU versus standard RT and BCNU for malignant glioma patients. J Neurooncol. 2018; 137: 39-47.

27. Hu X, Fang Y, Hui X, Jv Y, You C. Radiotherapy for diffuse brainstem glioma in children and young adults. Cochrane Database Syst Rev. 2016: Cd010439.

28. Câmara-Costa H, Resch A, Kieffer V, Lalande C, Poggi G, Kennedy C, et al. Neuropsychological Outcome of Children Treated for Standard Risk
Medulloblastoma in the PNET4 European Randomized Controlled Trial of Hyperfractionated Versus Standard Radiation Therapy and Maintenance Chemotherapy. Int J Radiat Oncol Biol Phys. 2015; 92: 978-85.

29. Cardinale R, Won M, Choucair A, Gillin M, Chakravarti A, Schultz C, et al. A phase II trial of accelerated radiotherapy using weekly stereotactic conformal boost for supratentorial glioblastoma multiforme: RTOG 0023. Int J Radiat Oncol Biol Phys. 2006; 65: 1422-8.

30. Shrieve DC, Alexander E, 3rd, Black PM, Wen PY, Fine HA, Kooy HM, et al. Treatment of patients with primary glioblastoma multiforme with standard postoperative radiotherapy and radiosurgical boost: prognostic factors and long-term outcome. J Neurosurg. 1999; 90: 72-7.

31. Reynaud T, Bertaut A, Farah W, Thibouw D, Crehange G, Truc G, et al. Hypofractionated Stereotactic Radiotherapy as a Salvage Therapy for Recurrent High-Grade Gliomas: Single-Center Experience. Technology in cancer research \& treatment. 2018; 17: 1533033818806498.

32. Roldan Urgoiti GB, Singh AD, Easaw JC. Extended adjuvant temozolomide for treatment of newly diagnosed glioblastoma multiforme. J Neurooncol. 2012; 108: 173-7.

33. Shah JL, Li G, Shaffer JL, Azoulay MI, Gibbs IC, Nagpal S, et al. Stereotactic Radiosurgery and Hypofractionated Radiotherapy for Glioblastoma. Neurosurgery. 2018; 82: 24-34.

34. Prestwich RJ, Sivapalasuntharam A, Johnston C, Evans K, Gerrard GE. Survival in high-grade glioma: a study of survival in patients unfit for or declining radiotherapy. Clin Oncol (R Coll Radiol). 2005; 17: 133-7.

35. Hegi ME, Diserens AC, Godard S, Dietrich PY, Regli L, Ostermann S, et al. Clinical trial substantiates the predictive value of O-6-methylguanine-DNA methyltransferase promoter methylation in glioblastoma patients treated with temozolomide. Clinical cancer research : an official journal of the American Association for Cancer Research. 2004; 10: 1871-4.

36. Esteller M, Garcia-Foncillas J, Andion E, Goodman SN, Hidalgo OF, Vanaclocha V, et al. Inactivation of the DNA-repair gene MGMT and the clinical response of gliomas to alkylating agents. N Engl J Med. 2000; 343: 1350-4.

37. Westphal M, Heese O, Steinbach JP, Schnell O, Schackert G, Mehdorn M, et al. A randomised, open label phase III trial with nimotuzumab, an anti-epidermal growth factor receptor monoclonal antibody in the treatment of newly diagnosed adult glioblastoma. Eur J Cancer. 2015; 51: 522-32.

38. Hart MG, Garside R, Rogers G, Stein K, Grant R. Temozolomide for high grade glioma. Cochrane Database Syst Rev. 2013: CD007415.

39. Jhaveri J, Liu Y, Chowdhary M, Buchwald ZS, Gillespie TW, Olson JJ, et al. Is less more? Comparing chemotherapy alone with chemotherapy and radiation for high-risk grade 2 glioma: An analysis of the National Cancer Data Base. Cancer. 2018; 124: 1169-78.

40. Perry JR, Laperriere N, O'Callaghan CJ, Brandes AA, Menten J, Phillips C, et al. Short-Course Radiation plus Temozolomide in Elderly Patients with Glioblastoma. N Engl J Med. 2017; 376: 1027-37.

41. Shaw EG, Wang M, Coons SW, Brachman DG, Buckner JC, Stelzer KJ, et al. Randomized trial of radiation therapy plus procarbazine, lomustine, and vincristine chemotherapy for supratentorial adult low-grade glioma: initial results of RTOG 9802. J Clin Oncol. 2012; 30: 3065-70.

42. Qian Y, Maruyama S, Kim H, Pollom EL, Kumar KA, Chin AL, et al. Cost-effectiveness of radiation and chemotherapy for high-risk low-grade glioma. Neuro Oncol. 2017; 19: 1651-60.

43. Wick W, Roth P, Hartmann C, Hau P, Nakamura M, Stockhammer F, et al. Long-term analysis of the NOA-04 randomized phase III trial of sequential radiochemotherapy of anaplastic glioma with PCV or temozolomide. Neuro Oncol. 2016; 18: 1529-37.

44. Jakacki RI, Cohen KJ, Buxton A, Krailo MD, Burger PC, Rosenblum MK, et al. Phase 2 study of concurrent radiotherapy and temozolomide followed by temozolomide and lomustine in the treatment of children with high-grade glioma: a report of the Children's Oncology Group ACNS0423 study. Neuro Oncol. 2016; 18: 1442-50.

45. Beier D, Proescholdt M, Reinert C, Pietsch T, Jones DTW, Pfister SM, et al. Multicenter pilot study of radiochemotherapy as first-line treatment for adults with medulloblastoma (NOA-07). Neuro Oncol. 2018; 20: 400-10.

46. Yamanaka R, Hayano A, Kanayama T. Radiation-induced gliomas: a comprehensive review and meta-analysis. Neurosurgical review. 2018; 41: 719-31.

47. Shein SA, Kuznetsov, II, Abakumova TO, Chelushkin PS, Melnikov PA, Korchagina AA, et al. VEGF- and VEGFR2-Targeted Liposomes for Cisplatin Delivery to Glioma Cells. Molecular pharmaceutics. 2016; 13: 3712-23.

48. Patel M, Vogelbaum MA, Barnett GH, Jalali R, Ahluwalia MS. Molecular targeted therapy in recurrent glioblastoma: current challenges and future directions. Expert opinion on investigational drugs. 2012; 21: 1247-66.

49. Scaringi C, Enrici RM, Minniti G. Combining molecular targeted agents with radiation therapy for malignant gliomas. Onco Targets Ther. 2013; 6: 1079_95.

50. Reardon DA, Turner S, Peters KB, Desjardins A, Gururangan S, Sampson JH, et al. A review of VEGF/VEGFR-targeted therapeutics for recurrent glioblastoma. J Natl Compr Canc Netw. 2011; 9: 414-27.

51. Yarden Y. The EGFR family and its ligands in human cancer. signalling mechanisms and therapeutic opportunities. Eur J Cancer. 2001; 37 Suppl 4: S3-8.

52. Kim MM, Umemura $Y$, Leung D. Bevacizumab and Glioblastoma: Past, Present, and Future Directions. Cancer journal (Sudbury, Mass). 2018; 24: $180-6$ 
53. Friedman HS, Prados MD, Wen PY, Mikkelsen T, Schiff D, Abrey LE, et al. Bevacizumab alone and in combination with irinotecan in recurrent glioblastoma. Journal of clinical oncology : official journal of the American Society of Clinical Oncology. 2009; 27: 4733-40.

54. Hasselbalch B, Lassen U, Hansen S, Holmberg M, Sørensen M, Kosteljanetz M, et al. Cetuximab, bevacizumab, and irinotecan for patients with primary glioblastoma and progression after radiation therapy and temozolomide: a phase II trial. Neuro Oncol. 2010; 12: 508-16.

55. Wang X, Guo G, Guan H, Yu Y, Lu J, Yu J. Challenges and potential of PD-1/PD-L1 checkpoint blockade immunotherapy for glioblastoma. Journal of experimental \& clinical cancer research : CR. 2019; 38: 87

56. Filley AC, Henriquez M, Dey M. Recurrent glioma clinical trial, CheckMate-143: the game is not over yet. Oncotarget. 2017; 8: 91779-94.

57. Sathornsumetee S, Desjardins A, Vredenburgh JJ, McLendon RE, Marcello J, Herndon JE, et al. Phase II trial of bevacizumab and erlotinib in patients with recurrent malignant glioma. Neuro Oncol. 2010; 12: 1300-10.

58. Bovi JA, Prah MA, Retzlaff AA, Schmainda KM, Connelly JM, Rand SD, et al. Pulsed Reduced Dose Rate Radiotherapy in Conjunction With Bevacizumab or Bevacizumab Alone in Recurrent High-grade Glioma: Survival Outcomes. International journal of radiation oncology, biology, physics. 2020; 108: 979-86.

59. Hofmann S, Schmidt MA, Weissmann T, Eyüpoglu I, Strnad A, Semrau S, et al. Evidence for improved survival with bevacizumab treatment in recurrent high-grade gliomas: a retrospective study with ("pseudo-randomized") treatment allocation by the health insurance provider. J Neurooncol. 2020; 148: 373-9.

60. Schwer AL, Damek DM, Kavanagh BD, Gaspar LE, Lillehei K, Stuhr K, et al. A phase I dose-escalation study of fractionated stereotactic radiosurgery in combination with gefitinib in patients with recurrent malignant gliomas. Int J Radiat Oncol Biol Phys. 2008; 70: 993-1001.

61. Lockhart AC, Rothenberg ML, Dupont J, Cooper W, Chevalier P, Sternas L, et al. Phase I study of intravenous vascular endothelial growth factor trap, aflibercept, in patients with advanced solid tumors. J Clin Oncol. 2010; 28: 207-14

62. de Groot JF, Lamborn KR, Chang SM, Gilbert MR, Cloughesy TF, Aldape K, et al. Phase II study of aflibercept in recurrent malignant glioma: a North American Brain Tumor Consortium study. J Clin Oncol. 2011; 29: 2689-95.

63. Bastien JI, McNeill KA, Fine HA. Molecular characterizations of glioblastoma, targeted therapy, and clinical results to date. Cancer. 2015; 121: 502-16.

64. Tivnan A, Heilinger T, Lavelle EC, Prehn JH. Advances in immunotherapy for the treatment of glioblastoma. J Neurooncol. 2017; 131: 1-9.

65. Goel G, Sun W. Cancer immunotherapy in clinical practice -- the past, present, and future. Chin J Cancer. 2014; 33: 445-57.

66. Herrera FG, Bourhis J, Coukos G. Radiotherapy combination opportunities leveraging immunity for the next oncology practice. CA: a cancer journal for clinicians. 2017; 67: 65-85

67. Sahebjam S, Sharabi A, Lim M, Kesarwani P, Chinnaiyan P. Immunotherapy and radiation in glioblastoma. J Neurooncol. 2017; 134: 531-9.

68. Lim M, Xia Y, Bettegowda C, Weller M. Current state of immunotherapy for glioblastoma. Nature reviews Clinical oncology. 2018; 15: 422-42.

69. Scheetz L, Kadiyala P, Sun X, Son S, Hassani Najafabadi A, Aikins M, et al. Synthetic High-density Lipoprotein Nanodiscs for Personalized Immunotherapy Against Gliomas. Clinical cancer research : an official journal of the American Association for Cancer Research. 2020; 26: 4369-80.

70. Pardoll DM. The blockade of immune checkpoints in cancer immunotherapy. Nature reviews Cancer. 2012; 12: 252-64

71. Reznik E, Smith AW, Taube S, Mann J, Yondorf MZ, Parashar B, et al. Radiation and Immunotherapy in High-grade Gliomas: Where Do We Stand? American journal of clinical oncology. 2018; 41: 197-212.

72. Berghoff AS, Kiesel B, Widhalm G, Rajky O, Ricken G, Wöhrer A, et al. Programmed death ligand 1 expression and tumor-infiltrating lymphocytes in glioblastoma. Neuro Oncol. 2015; 17: 1064-75.

73. Parsa AT, Waldron JS, Panner A, Crane CA, Parney IF, Barry JJ, et al. Loss of tumor suppressor PTEN function increases B7-H1 expression and immunoresistance in glioma. Nature medicine. 2007; 13: 84-8.

74. Vega EA, Graner MW, Sampson JH. Combating immunosuppression in glioma. Future oncology (London, England). 2008; 4: 433-42

75. Qian BZ, Pollard JW. Macrophage diversity enhances tumor progression and metastasis. Cell. 2010; 141: 39-51.

76. Zhu C, Kros JM, Cheng C, Mustafa D. The contribution of tumor-associated macrophages in glioma neo-angiogenesis and implications for anti-angiogenic strategies. Neuro Oncol. 2017; 19: 1435-46.

77. Dai H, Wang $\mathrm{Y}$, Lu X, Han W. Chimeric Antigen Receptors Modified T-Cells for Cancer Therapy. J Natl Cancer Inst. 2016; 108: djv439.

78. Zeng J, See AP, Phallen J, Jackson CM, Belcaid Z, Ruzevick J, et al. Anti-PD-1 blockade and stereotactic radiation produce long-term survival in mice with intracranial gliomas. Int J Radiat Oncol Biol Phys. 2013; 86: 343-9.

79. Binder DC, Davis AA, Wainwright DA. Immunotherapy for cancer in the central nervous system: Current and future directions. Oncoimmunology. 2016; 5: e1082027.

80. Dovedi SJ, Adlard AL, Lipowska-Bhalla G, McKenna C, Jones S, Cheadle EJ, et al. Acquired resistance to fractionated radiotherapy can be overcome by concurrent PD-L1 blockade. Cancer research. 2014; 74: 5458-68.

81. Giladi M, Munster M, Schneiderman RS, Voloshin T, Porat Y, Blat R, et al. Tumor treating fields (TTFields) delay DNA damage repair following radiation treatment of glioma cells. Radiation oncology (London, England). 2017; 12: 206.

82. Stupp R, Taillibert S, Kanner A, Read W, Steinberg D, Lhermitte B, et al. Effect of Tumor-Treating Fields Plus Maintenance Temozolomide vs Maintenance Temozolomide Alone on Survival in Patients With Glioblastoma: A Randomized Clinical Trial. Jama. 2017; 318: 2306-16.

83. Gutin PH, Wong ET. Noninvasive application of alternating electric fields in glioblastoma: a fourth cancer treatment modality. American Society of Clinical Oncology educational book American Society of Clinical Oncology Annual Meeting. 2012: 126-31.

84. Giladi M, Schneiderman RS, Voloshin T, Porat Y, Munster M, Blat R, et al. Mitotic Spindle Disruption by Alternating Electric Fields Leads to Improper Chromosome Segregation and Mitotic Catastrophe in Cancer Cells. Scientific reports. 2015; 5: 18046

85. Neuhaus E, Zirjacks L, Ganser K, Klumpp L, Schüler U, Zips D, et al. Alternating Electric Fields (TTFields) Activate Ca(v)1.2 Channels in Human Glioblastoma Cells. Cancers. 2019; 11:110.

86. Gera N, Yang A, Holtzman TS, Lee SX, Wong ET, Swanson KD. Tumor treating fields perturb the localization of septins and cause aberrant mitotic exit. PloS one. 2015; 10: e0125269.

87. Wenger C, Salvador R, Basser PJ, Miranda PC. Improving Tumor Treating Fields Treatment Efficacy in Patients With Glioblastoma Using Personalized Array Layouts. Int J Radiat Oncol Biol Phys. 2016; 94: 1137-43.

88. Ballo MT, Urman N, Lavy-Shahaf G, Grewal J, Bomzon Z, Toms S. Correlation of Tumor Treating Fields Dosimetry to Survival Outcomes in Newly Diagnosed Glioblastoma: A Large-Scale Numerical Simulation-Based Analysis of Data from the Phase 3 EF-14 Randomized Trial. Int J Radiat Oncol Biol Phys. 2019: 104: 1106-13.

89. Taphoorn MJB, Dirven L, Kanner AA, Lavy-Shahaf G, Weinberg U, Taillibert $\mathrm{S}$, et al. Influence of Treatment With Tumor-Treating Fields on Health-Related Quality of Life of Patients With Newly Diagnosed Glioblastoma: A Secondary Analysis of a Randomized Clinical Trial. JAMA Oncol. 2018; 4: 495-504

90. Zhu JJ, Demireva P, Kanner AA, Pannullo S, Mehdorn M, Avgeropoulos N, et al. Health-related quality of life, cognitive screening, and functional status in a randomized phase III trial (EF-14) of tumor treating fields with temozolomide compared to temozolomide alone in newly diagnosed glioblastoma. J Neurooncol. 2017; 135: 545-52.

91. Lazaridis L, Schäfer N, Teuber-Hanselmann S, Blau T, Schmidt T, Oster C, et al. Tumour Treating Fields (TTFields) in combination with lomustine and temozolomide in patients with newly diagnosed glioblastoma. J Cancer Res Clin Oncol. 2020; 146: 787-92.

92. Lu G, Rao M, Zhu P, Liang B, El-Nazer RT, Fonkem E, et al. Triple-drug Therapy With Bevacizumab, Irinotecan, and Temozolomide Plus Tumor Treating Fields for Recurrent Glioblastoma: A Retrospective Study. Frontiers in neurology. 2019; 10: 42

93. Hottinger AF, Pacheco P, Stupp R. Tumor treating fields: a novel treatment modality and its use in brain tumors. Neuro Oncol. 2016; 18: 1338-49.

94. Onken J, Staub-Bartelt F, Vajkoczy P, Misch M. Acceptance and compliance of TTFields treatment among high grade glioma patients. J Neurooncol. 2018; 139: $177-84$.

95. Sutera P, Kalash R, Flickinger J, Engh J, Heron DE. Clinical and Molecular Recursive Partitioning Analysis of High-grade Glioma Treated With IMRT. American journal of clinical oncology. 2019; 42: 27-35.

96. Carlson JA, Reddy K, Gaspar LE, Ney D, Kavanagh BD, Damek D, et al. Hypofractionated-intensity modulated radiotherapy (hypo-IMRT) and temozolomide (TMZ) with or without bevacizumab (BEV) for newly diagnosed glioblastoma multiforme (GBM): a comparison of two prospective phase II trials. J Neurooncol. 2015; 123: 251-7.

97. Zhong L, Chen L, Lv S, Li O, Chen G, Luo W, et al. Efficacy of moderately hypofractionated simultaneous integrated boost intensity-modulated radiotherapy combined with temozolomide for the postoperative treatment of glioblastoma multiforme: a single-institution experience. Radiation oncology (London, England). 2019; 14: 104

98. Reddy K, Damek D, Gaspar LE, Ney D, Waziri A, Lillehei K, et al. Phase II trial of hypofractionated IMRT with temozolomide for patients with newly diagnosed glioblastoma multiforme. Int J Radiat Oncol Biol Phys. 2012; 84: 655-60.

99. Monjazeb AM, Ayala D, Jensen C, Case LD, Bourland JD, Ellis TL, et al. A phase I dose escalation study of hypofractionated IMRT field-in-field boost for newly diagnosed glioblastoma multiforme. Int J Radiat Oncol Biol Phys. 2012; 82: $743-8$.

100. Jastaniyah N, Murtha A, Pervez N, Le D, Roa W, Patel S, et al. Phase I study of hypofractionated intensity modulated radiation therapy with concurrent and adjuvant temozolomide in patients with glioblastoma multiforme. Radiation oncology (London, England). 2013; 8: 38.

101. Cloughesy TF, Mochizuki AY, Orpilla JR, Hugo W, Lee AH, Davidson TB, et al. Neoadjuvant anti-PD-1 immunotherapy promotes a survival benefit with intratumoral and systemic immune responses in recurrent glioblastoma. Nature medicine. 2019; 25: 477-86.

102. Desjardins A, Gromeier M, Herndon JE, 2nd, Beaubier N, Bolognesi DP, Friedman $\mathrm{AH}$, et al. Recurrent Glioblastoma Treated with Recombinant Poliovirus. N Engl J Med. 2018; 379: 150-61.

103. Brown CE, Alizadeh D, Starr R, Weng L, Wagner JR, Naranjo A, et al. Regression of Glioblastoma after Chimeric Antigen Receptor T-Cell Therapy. N Engl J Med. 2016; 375: 2561-9. 
104. O'Rourke DM, Nasrallah MP, Desai A, Melenhorst JJ, Mansfield K, Morrissette JJD, et al. A single dose of peripherally infused EGFRvIII-directed CAR T cells mediates antigen loss and induces adaptive resistance in patients with recurrent glioblastoma. Science translational medicine. 2017; 9: eaaa0984.

105. Hilf N, Kuttruff-Coqui S, Frenzel K, Bukur V, Stevanović S, Gouttefangeas C, et al. Actively personalized vaccination trial for newly diagnosed glioblastoma. Nature. 2019; 565: 240-5.

106. Toms SA, Kim CY, Nicholas G, Ram Z. Increased compliance with tumor treating fields therapy is prognostic for improved survival in the treatment of glioblastoma: a subgroup analysis of the EF-14 phase III trial. J Neurooncol. 2019; 141: 467-73.

107. Kirson ED, Dbalý V, Tovarys F, Vymazal J, Soustiel JF, Itzhaki A, et al. Alternating electric fields arrest cell proliferation in animal tumor models and human brain tumors. Proceedings of the National Academy of Sciences of the United States of America. 2007; 104: 10152-7.

108. Kirson ED, Schneiderman RS, Dbalý V, Tovarys F, Vymazal J, Itzhaki A, et al. Chemotherapeutic treatment efficacy and sensitivity are increased by adjuvant alternating electric fields (TTFields). BMC medical physics. 2009; 9: 1.

109. Stupp R, Taillibert S, Kanner AA, Kesari S, Steinberg DM, Toms SA, et al. Maintenance Therapy With Tumor-Treating Fields Plus Temozolomide vs Temozolomide Alone for Glioblastoma: A Randomized Clinical Trial. Jama. 2015; 314: 2535-43.

110. Mrugala MM, Engelhard HH, Dinh Tran D, Kew Y, Cavaliere R, Villano JL, et al. Clinical practice experience with NovoTTF-100A $\mathrm{A}^{\mathrm{TM}}$ system for glioblastoma: The Patient Registry Dataset (PRiDe). Seminars in oncology. 2014; 41 Suppl 6: S4-s13.

111. Stupp R, Wong ET, Kanner AA, Steinberg D, Engelhard H, Heidecke V, et al. NovoTTF-100A versus physician's choice chemotherapy in recurrent glioblastoma: a randomised phase III trial of a novel treatment modality. Eur J Cancer. 2012; 48: 2192-202. 